\title{
The avian collection of the former Saint Augustine College of the Regional Natural History Museum in Plovdiv
}

\author{
Zlatozar Boev ${ }^{1}$, Ognyan Todorov ${ }^{2}$
}

1 National Museum of Natural History, Bulgarian Academy of Sciences, 1 Tsar Osvoboditel Blvd, 1000 Sofia, Bulgaria, boev@nmnhs.com, zlatozarboev@gmail.com

2 Regional Natural History Museum in Plovdiv, 34 Hristo Danov Street, 4000 Plovdiv, Bulgaria, ogi_lion@abv.bg

\begin{abstract}
The paper presents a catalogue of bird specimens (dry mounted preserves) survived after the closing (1948) of the former Catholic College "Saint Augustine". Currently, the studied specimens are kept at the Regional Natural History Museum in Plovdiv. The collection includes a total of 193 species of birds of 81 families and 28 orders, represented by 205 specimens (circa two percent of the recent World avifauna). Over 15 percent of the species have an IUCN conservation status (NT to CR). The most valuable are the following specimens: critically endangered grey-backed myna (Acridotheres tricolor) and two endangered species - echo parakeet (Psittacula eques) and sooty albatross (Phoebetria fusca). This is the $2^{\text {nd }}$ largest collection of exotic birds in Bulgaria.
\end{abstract}

Keywords: avian collections, rare bird specimens, natural history museums, avian rarities

\section{Introduction}

The former catholic college "Saint Augustine" (known also as the French College; Le collège St. Augustine, Philippopolis; Fig. 1) operated at the end of $19^{\text {th }}$ and the first decades of $20^{\text {th }}$ centuries (1884-1948). The historian Augustine Travernier (Brother Boris; 18601928) founded a pedagogical museum at the college. After 1898, both he and Hermann Gisler collected many items and exhibits for the museum. The college was closed in 1948 and its collections were transferred to the newly established History Museum of Plovdiv (1952) and the Plovdiv University in 1951 (Todorov, 2016; Georgiev, 2017). The Museum was open for visitors on 5 September 1955 (Angelov, 1957).

Bird collection of the total mounted dry exhibits represented a valuable integral part of the natural history collections of the French College. In its Department of Birds mounted bird specimens have been kept along with eggs, nests and skeletons of birds (Todorov, 2016).

Unfortunately, when the college was closed, labels of the exhibits were lost in their transportation and transferred to the buildings of other institutions. Thus, the scientific value of this second important Bulgarian collection of exotic birds (after the collections of the National Museum of Natural History in Sofia; Boev, 1991, 2003) was compromised due to its incompetent treating. Until recently (2012), these specimens had been kept at the fund repositories of the Regional Natural History Museum in Plovdiv and they had not been exposed to visitors in the exhibition halls (Todorov, 2016). A considerable part of them was damaged - bills, tails, legs or wings were broken, plumage was damaged by moths. After the complete reconstruction of the museum building, this collection has attracted the attention of the present director of the museum, who started a gradual restoration of its specimens. In 2016, a significant part of them (100 specimens) were returned again in the show-cases in the exhibition halls of the Museum (Todorov, 2016).

So far, there are no publications describing themuseum's ornithological collection. The only publication deals with specimens of Falconiformes and Pelecaniformes (Petrov, 1983) and states that the museum holds about 50 specimens (of about 30 species) of foreign birds, which are inherited from the former French College in Plovdiv, for which there is no "sci- 


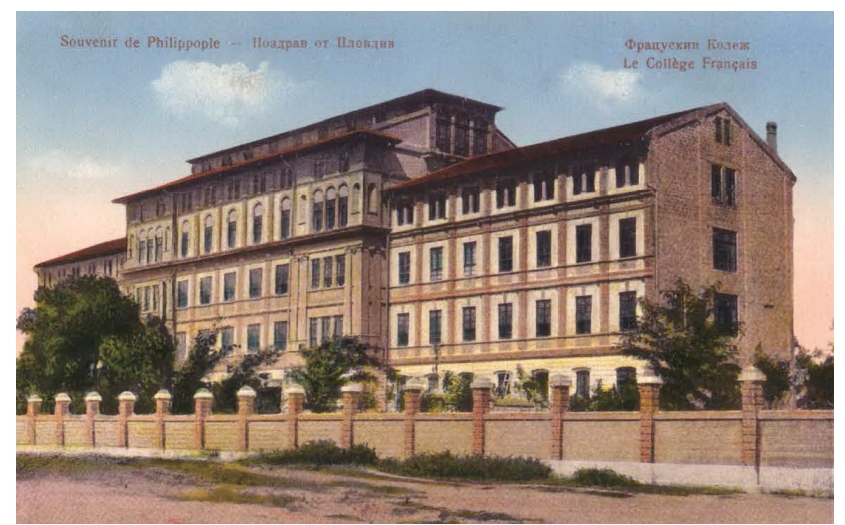

Fig. 1. The building of the French College Saint Augustine in Plovdiv, last decades of 19 th century.

entific" (i.e. collector's) data and have only exhibition value.

The published information of Roselaar (2003) (provided by Zlatozar Boev) is also rather laconic: "[The Museum] Includes the former coll. of the College St. Augustine (Philipopol), obtained 1955. Includes 662 skins (250 species), largely from S Bulgaria." As seen bellow, only the present collection of exotic birds includes 193 species.

In this paper, we present a complete list of all survived specimens of bird preserves of the French College.

A very small part of the former bird collection of the French College (six specimens) survived and still exist in the "St. Cyril and Methodius" High School in Plovdiv. All they are listed in the paper (see below).

\section{Material and methods}

The survived specimens of the collection belong to 193 species of mostly exotic wild birds of European, Asian, North and South American and Astralasian fauna. It does not include either domestic forms or decorative birds kept as pets. All specimens have normal plumage typical coloration for the relevant species/sex. There are no specimens of abnormal development of bill, legs, etc.

Taxonomy follows del Hoyo \& Collar (2014, 2016).

For each specimen after the scientific and English names are given its sex and age (wherever possible), IUCN conservation status, as well as its inventory numbers of the Regional Natural History Museum in Plovdiv and the former French Catholic College "Saint Augustine", although many specimens lack these numbers.

Abbreviations: (1) Institutional: FCSA - French College "Saint Augustine"; CMHSP - "St Cyril and Methodius" High School in Plovdiv; RNHMP - Regional Natural History Museum, Plovdiv] (2) Conservational: CR - critically endangered; EN - endangered; LC - least concern; NT - near threatened; VU - vulnerable.

\section{Systematic list}

Order Rheiformes

Family Rheidae

- Rhea americana Linnaeus 1758 greater rhea. - ad. NT (RNHMP 990; FCSA 258).

Family Casuariidae

- Dromaius novaehollandiae Latham, 1790 common emu. - juv. LC (RNHMP 977; FCSA 218).

Order Galliformes

Family Phasianidae

- Chrysolophus pictus (Linnaeus, 1758) golden pheasant. - $\hat{\sigma}$ ad. LC (RNHMP 274).

- Crossoptilon mantchuricum Swinhoe, 1863 brown eared-pheasant. - ô ad. VU (RNHMP 834).

- Crossoptilon auritum (Pallas, 1811) blue earedpheasant. - ad. LC (RNHMP 879).

- Melanoperdix niger (Vigors, 1829) black partridge. - $q$ ad. VU (RNHMP 978; FCSA 256).

- Syrmaticus ellioti (Swinhoe, 1872) Elliot's pheasant. $-\widehat{O}$ ad. NT (RNHMP 836).

Order Anseriformes

Family Anatidae

- Anser cygnoides (Linnaeus, 1758) swan goose. ad. VU (RNHMP 578).

- Aix sponsa (Linnaeus, 1758) wood duck. - $\delta$ ad. LC (RNHMP 858; FCSA 77) 
The avian collection of the former Saint Augustine College of the Regional Natural History Museum in Plovdiv

- Bucephala albeola (Linnaeus, 1758) bufflehead. o ad. LC (RNHMP 835; FCSA 132, 71).

- Dendrocygna bicolor (Vieillot, 1816) fulvous whistling-duck. - ad. LC (RNHMP 865; FCSA 56).

- Dendrocygna javanica (Horsfield, 1821) lesser whistling-duck. - ad. LC (RNHMP 837; FCSA 66).

- Dendrocygna viduata (Horsfield, 1821) whitefaced whistling-duck. - ad. LC (RNHMP 829; FCSA 76).

- Somateria spectabilis (Linnaeus, 1758) king eider. - $\widehat{o}$ ad. LC (RNHMP 979; FCSA 35).

- Clangula hyemalis (Linnaeus, 1758) long-tailed duck. - ô ad. VU (RNHMP 880; FCSA 67).

- Alopochen aegyptiacus (Linnaeus, 1766) egyptian goose. - ad. LC (RNHMP 1033; FCSA 40).

Order Podicipediformes

Family Podicipedidae

- Podiceps grisegena (Boddaert, 1783) red-necked grebe. - juv. LC (RNHMP 856; FCSA 62, 207).

- Podilymbus podiceps (Linnaeus, 1758) pied-billed grebe. - ad. LC (RNHMP 958; FCSA 79).

Order Columbiformes

Family Columbidae

- Claravis pretiosa (Ferrari-Perez, 1886) blue ground-dove. - $\hat{\jmath}$ ad. LC (RNHMP 773; FCSA 79).

- Zenaida macroura (Linnaeus, 1758) mourning dove. - ô ad. LC (RNHMP 774; FCSA 245).

- Spilopelia senegalensis (Linnaeus, 1766) laughing dove. - ad. LC (RNHMP 1023; FCSA 247).

- Streptopelia turtur (Linnaeus, 1758) European turtle-dove. - ad. LC (RNHMP 1022; FCSA 250).

Order Pterocliformes

Family Pteroclididae

- Pterocles namaqua (Gmelin, 1789) Namaqua sandgrouse. - + ad. LC (RNHMP 1024, 1025; FCSA 1).
- Pterocles exustus olivascens (Hartert, 1909) chestnut-bellied sandgrouse. - ô ad. LC (RNHMP 1025; FCSA 2).

Order Caprimulgiformes

Family Trochilidae

- Amazilia fimbriata Gmelin, 1788 glittering-throated emerald. - ad. LC (RNHMP 813).

- Campylopterus cuvierii (Dellatre \& Bourcier, 1846) scaly-breasted sabrewing. - ad. LC (RNHMP 925).

- Campylopterus duidae duidae Chapman, 1929 buff-breasted sabrewing. - ad. LC (RNHMP 814).

- Campylopterus duidae guaiquinimae Zimmer \& Phelps, 1946 buff-breasted sabrewing. - ad. LC (RNHMP 912).

- Clytolaema rubricauda (Boddaert, 1783) Brazilian ruby. - $\hat{\sigma}$ ad. LC (RNHMP 921).

- Cyanophaia bicolor (Gmeilin, 1788) blue-headed hummingbird. - ô ad. LC (RNHMP 917).

- Eugenes fulgens (Swainson, 1827) magnificent hummingbird. - + ad. LC (RNHMP 913).

- Eupetomena macroura macroura (J. F. Gmelin, 1788) swallow-tailed hummingbird. - ad. LC (RNHMP 918).

- Heliangelus strophianus (Gould, 1846) gorgeted sunangel. - $\hat{\sigma}$ ad. LC (RNHMP 919).

- Lesbia victoriae Bourcier \& Muslant, 1846 blacktailed trainbearer. - $q$ ad. LC (RNHMP 923).

- Leucippus fallax (Bourcier, 1843) buffy hummingbird. - $\hat{o}$ ad. LC (RNHMP 911).

- Lophornis ornatus (Boddaert, 1783) tufted coquette. - §o ad. LC (RNHMP 916).

- Phaethornis guy (Lesson, 1833) green hermit. - $\widehat{\sigma}$ ad. LC (RNHMP 924).

- Polyonymus caroli Bourcier, 1847 bronze-tailed comet. - §o ad. LC (RNHMP 915).

- Chrysolampis mosquitus (Linnaeus, 1758) rubytopaz hummingbird. - ô ad. LC (RNHMP 926).

Order Opisthocomiformes

Family Opisthocomidae

- Opisthocomus hoazin (Müller, 1776) hoatzin. ad. LC (RNHMP 980; FCSA 145). 
Order Cuculiformes

Family Cuculidae

- Centropus anselli Sharpe, 1874 Gabon coucal. ad. LC (RNHMP 973; FCSA 87).

- Centropus celebensis Quoy \& Gaimard, 1830 bay coucal. - ad. LC (RNHMP 974; FCSA 72).

- Centropus unirufus (Cabanis \& Heine, 1863) rufous coucal. - ad. NT (RNHMP 974; FCSA 9, 149).

- Eudynamys scolopaceus (Linnaeus, 1758) western koel. - 9 ad. LC (RNHMP 970; FCSA 131); (RNHMP 779).

- Tapera naevia (Linnaeus, 1766) striped cuckoo. ad. LC (RNHMP 848).

Order Gruiformes

Family Rallidae

- Aramides ypecaha Vieillot, 1819 giant wood-rail. - ad. LC (RNHMP 833; FCSA 246).

- Pardirallus sanguinolentus (Swainson, 1838) plumbeous rail. - ad. LC (RNHMP 961; FCSA 248).

- Zapornia flavirostra (Swainson, 1837) black crake. - ad. LC (RNHMP 812; FCSA 248).

Order Musophagiformes

Family Musophagidae

- Tauraco livingstonii (Gray, 1864) Livingstne's turaco. - ad. LC (RNHMP 804).

Order Sphenisciformes

Family Spheniscidae

- Spheniscus humboldti Meyen, 1834 Humboldt penguin. - ad. VU (RNHMP 589); (RNHMP 584); (RNHMP 723).

Order Procelllariiformes

Family Diomedeidae

- Phoebetria fusca Hilsenberg, 1822 sooty albatross. - ad. EN (RNHMP 855) (Fig. 2).

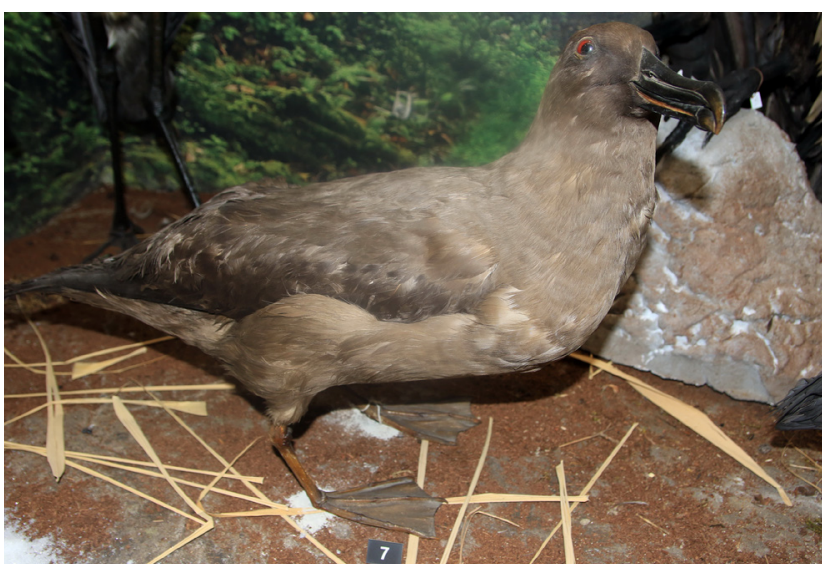

Fig. 2. Sooty albatross (Phoebetria fusca), RNHMP 855. Photograph: Ognyan Todorov.

Family Procellariidae

- Daption capense (Linnaeus, 1758) Cape petrel. ad. LC (RNHMP 908, 854).

Order Ciconiiformes

Family Ciconiidae

- Leptoptilos crumenifer (Lesson, 1831) marabou. - ad. LC (RNHMP 828)

Order Pelecaniformes

Family Threskiornithidae

- Eudocimus ruber (Linnaeus, 1758) scarlet ibis. ad. LC (RNHMP 824, FCSA 216); (RNHMP 981, FCSA 252); (RNHMP 982; FCSA 240).

- Platalea ajaja Linnaeus, 1758 roseate spoonbill. - ad. LC (RNHMP 955)

- Plegadis falcinellus Linnaeus, 1766 glossy ibis. ad. LC (CMHSP).

- Theristicus caudatus (Boddaert, 1783) blackfaced ibis. - ad. LC (RNHMP 827).

Family Ardeidae

- Butorides striata virescens (Linnaeus, 1758) green-backed heron. - ad. LC (RNHMP 946; FCSA 231); (RNHMP 1001).

- Cochlearius cochlearius (Linnaeus, 1766) boatbilled heron. - ad. LC (RNHMP 823; FCSA 256). 
The avian collection of the former Saint Augustine College of the Regional Natural History Museum in Plovdiv

- Ardeola ralloides (Scopoli, 1769) squacco heron. - ad. LC (CMHSP).

- Egretta rufescens (Gmelin, 1789) reddish egret. ad. NT (RNHMP 825).

- Nyctanassa violacea (Linnaeus, 1758) yellowcrowned night-heron. - ad. LC (RNHMP 822; RNHMP 983).

- Ardea melanocephala (Vigors \& Children, 1826) black-headed heron. - juv. LC (RNHMP 826).

Order Phaethontiformes

Family Phaethontidae

- Phaethon lepturus lepturus Daurin, 1802 whitetailed tropicbird. - ad. LC (RNHMP 1003; FCSA 11).

Order Suliformes

Family Phalacrocoracidae

- Microcarbo africanus (Gmelin, 1789) long-tailed cormorant. - ad. LC (RNHMP 857; FCSA 57).

Family Anhingidae

- Anhinga rufa (Daurin, 1802) African darter. - ad. LC (RNHMP 708).

Order Charadriiformes

Family Pluvianidae

- Pluvianus eagyptius (Linnaeus, 1758) Egyptian plover. - ad. LC (RNHMP 756, FCSA 85; RNHMP 959; FCSA 99).

Family Haematopodidae

- Haematopus ater Vieillot \& Oudart, 1825 blackish oystercatcher. - ad. LC (RNHMP 858).

Family Charadriidae

- Vanellus spinosus (Linnaeus, 1758) spur-winged lapwing. - ad. LC (RNHMP 860; FCSA 215).

- Vanellus tectus (Boddaert, 1783) black-headed lapwing. - ad. LC (RNHMP 960; FCSA 238).
- Vanellus vanellus (Linnaeus, 1758) northern lapwing. - ad. LC (RNHMP 834).

Family Scolopacidae

- Numenius arquata (Linnaeus, 1758) Eurasian curlew. - ad. NT (CMHSP).

Family Jacanidae

- Actophilornis africanus (Gmelin, 1789) African jacana. - ad. LC (RNHMP 832; FCSA 225).

- Jacana spinosa (Linnaeus, 1758) northern jacana. - ad. LC (RNHMP 831; FCSA 23).

Family Turnicidae

- Turnix sylvaticus Desfontaines, 1789 common buttonquail. - ad. $\rightarrow$ ad. LC (RNHMP 1026; FCSA 259); ad. (RNHMP 984).

Family Laridae

- Hydroprogne caspia (Pallas, 1770) Caspian tern. - ad. LC (RNHMP 985; FCSA 235).

Family Stercorariidae

- Catharacta antarctica (Lesson, 1831) brown skua. - ad. LC (RNHMP 853; FCSA 446).

Family Alcidae

- Alle alle (Linnaeus, 1758) little auk. - ad. LC (RNHMP 950, FCSA 239; RNHMP 951, FCSA 21?; RNHMP 752; FCSA 233).

- Cepphus grylle (Linnaeus, 1758) black guillemot. - ad. LC (RNHMP 859; FCSA 212).

Order Strigiformes

Family Strigidae

- Otus senegalensis (Swainson, 1837) African scops-owl. - ad. LC (RNHMP 963).

- Strix aluco Linnaeus, 1758 tawny owl. - ad. LC (CMHSP). 
Order Cathartiformes

Family Cathartidae

- Vultur gryphus Linnaeus, 1758 Andean condor. ad. NT (RNHMP 456).

Order Accipitriformes

Family Accipitridae

- Aquila rapax (Temminck, 1828) tawny eagle. ad. LC (CMHSP).

- Buteo rufinus Cretzchmar, 1829 long-legged buzzard. - ad. LC (RNHMP 844; FCSA 330).

- Buteo platypterus Vieillot, 1823 broad-winged hawk. - ad. LC (CMHSP).

- Pernis ptilorhynchus torquatus Lesson, 1831 oriental honey-buzzard. - ad. LC (RNHMP 847; FCSA 237).

Order Coliiformes

Family Coliidae

- Urocolius macrourus (Linnaeus, 1766) bluenaped mousebird. - ad. LC (RNHMP 1012; FCSA 190).

Order Bucerotiformes

Family Bucerotidae

- Lophoceros pallidirostris (Hartlaub \& Finsch, 1870) pale-billed hornbill. - ad. LC (RNHMP 733; FCSA 19).

- Lophoceros semifasciatus Chapman, 1922 West African pied hornbill. - ad. LC (RNHMP 750).

- Tockus erythrorhynchus Temminck, 1823 redbilled hornbill. - ad. LC (RNHMP 734).

Order Coraciiformes

Family Meropidae

- Merops pusillus Statius Muller, 1776 little beeeater. - ad. LC (RNHMP 776; FCSA 111 A71).
Family Coraciidae

- Coracias cyanogaster Cuvier, 1816 blue-bellied roller. - ad. LC (RNHMP 805), (RNHMP 986; FCSA 103).

- Eurystomus glaucurus (Statius Muller, 1776) broad-billed roller. - ad. LC (RNHMP 781; FCSA 117).

Family Momotidae

- Momotus momota (Linnaeus, 1766) Amazonian motmot. - ad. LC (RNHMP 743; FCSA 83).

Family Alcedinidae

- Ceryle rudis (Linnaeus, 1758) pied kingfisher. ad. LC (RNHMP 798).

- Megaceryle torquata (Linnaeus, 1766) ringed kingfisher. - ad. LC (RNHMP 797).

- Pelargopsis capensis burmanica (Sharpe, 1870 stork-billed kingfisher. - ad. LC (RNHMP 799).

Order Piciformes

Family Bucconidae

- Nystactes tamatia Gmelin, 1788 spotted puffbird. - ad. LC (RNHMP 806; FCSA 199 B31).

- Malacoptila striata (Spix, 1824) greater crescentchested puffbird. - ad. NT (RNHMP 971; FCSA 98).

Family Ramphastidae

- Pteroglossus inscriptus Swainson, 1822 lettered aracari. - ad. LC (RNHMP 803).

- Pteroglossus torquatus (Gmelin, 1788) collared aracari. - ad. LC (RNHMP 801).

- Ramphastos tucanus Linnaeus, 1758 red-billed toucan. - ad. VU (RNHMP 987; FCSA 185).

- Ramphastos vitellinus Lichtenstein, 1823 channel-billed toucan. - ad. VU (RNHMP 802; FCSA 196).

Family Capitonidae

- Capito niger (Statius Müller, 1776) black-spotted barbet. - ad. LC (RNHMP 967; FCSA 186). 
The avian collection of the former Saint Augustine College of the Regional Natural History Museum in Plovdiv

Family Lybiidae

- Lybius bidentatus (Shaw, 1798) double-toothed barbet. - ad. LC (RNHMP 815).

Order Falconiformes

Family Falconidae

- Microhierax melanoleucos (Blyth, 1843) pied falconet. - ad. LC (RNHMP 839).

Order Psittaciformes

Family Psittacidae

- Eolophus roseicapilla (Vieillot, 1817) galah. - ad. LC (RNHMP 770).

- Ara militaris (Linnaeus, 1766) military macaw. ad. VU (RNHMP 769).

- Platycercus eximius (Shaw, 1792) eastern rosella. - ad. LC (RNHMP 915).

- Psittacula eques (Boddaert, 1783) echo parakeet. - $q$ ad. EN (RNHMP 988; FCSA 69) (Fig. 3).

- Psittacula krameri (Scopoli, 1769) rose-ringed parakeet. - ad. LC (RNHMP 920); (RNHMNP 921); (RNHMP 989).

- Melopsittacus undulatus (Shaw, 1805) budgerigar. - ad. LC (RNHMP 968).

- Pyrrhura molinae Massena \& Souance, 1854 green-cheeked parakeet. - ad. LC (RNHMP 772).

Order Passeriformes

Family Eurylaimidae

- Cymbirhynchus macrorhynchos (Gmelin, 1783) black-and-red broadbill. - $\hat{\sigma}$ ad. LC (RNHMP 815; FCSA 219).

Family Thamnophilidae

- Pyriglena leuconota castanoptera Chubb, 1916 white-backed fire-eye. - $q$ ad. LC (RNHMP 956; FCSA 195)

- Pithys albifrons (Linnaeus, 1766) white-plumed antbird. - ad. LC (RNHMP 793).

- Mackenziaena leachii (Such, 1825) large-tailed antshrike. - ô ad. LC (RNHMP 1021; FCSA 86).

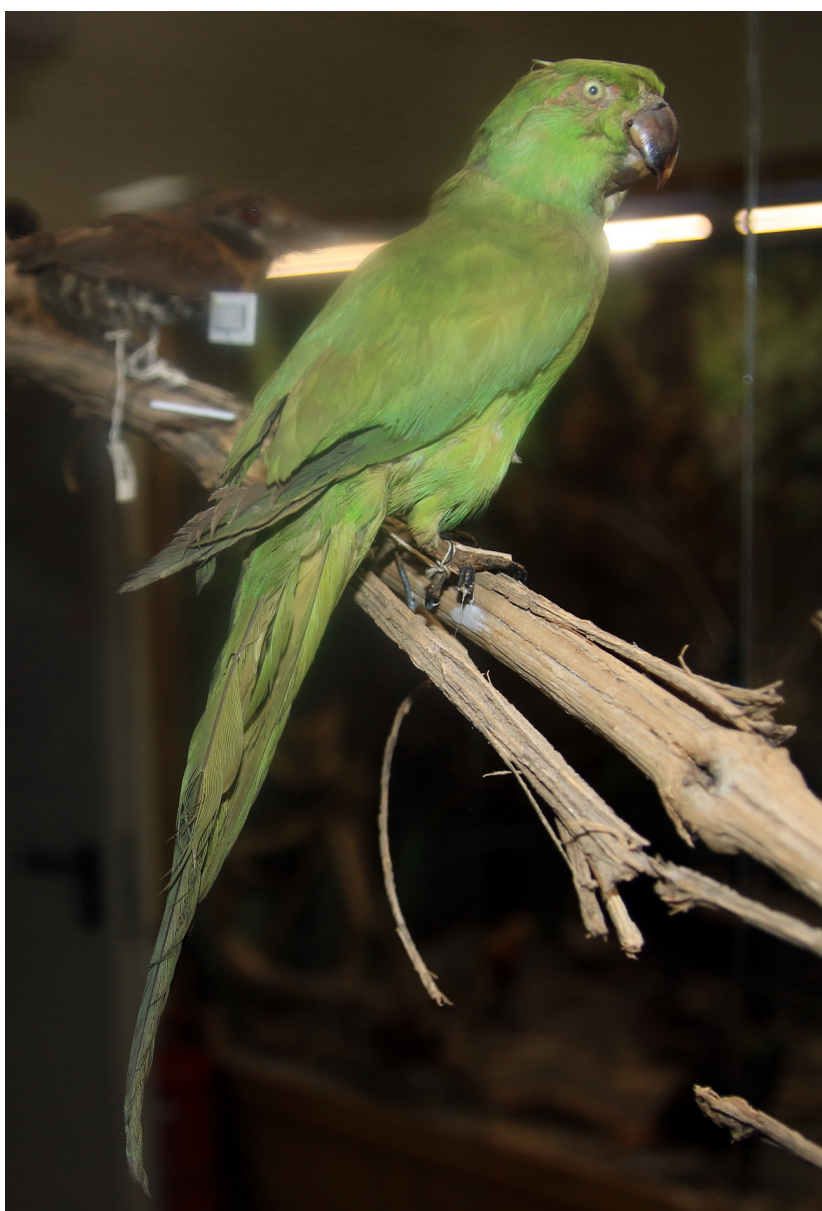

Fig. 3. Echo parakeet (Psittacula eques), RNHMP 988. Photograph: Ognyan Todorov.

Family Furnariidae

- Dendrocolaptes picumnus Lichtenstein, 1820 black-banded woodcreeper. - ad. LC (RNHMP 789; FCSA 163).

Family Pipridae

- Chiroxiphia pareola (Linnaeus, 1766) bluebacked manakin. - ad. LC (RNHMP 768).

- Carpornis cucullata (Swainson, 1821) hooded berryeater. - ad. NT (RNHMP 809; FCSA - 67).

- Pseudopipra pipra (Linnaeus, 1758) whitecrowned manakin. - ad. LC (RNHMP 843; FCSA 48). 
Family Cotingidae

- Gymnoderus foetidus foetidus (Linnaeus, 1758) bare-necked fruitcrow. - ad. LC (RNHMP 976; FCSA 18).

- Haematoderus militaris Bonaparte, 1854 crimson fruitcrow. - ad. LC (RNHMP 975; FCSA 129).

- Rupicola rupicola (Linnaeus, 1766) Guianan cock-of-the-rock. - ad. LC (RNHMP 952).

- Procnias nudicollis (Vieillot, 1817) bare-throated bellbird. - ô ad. VU (RNHMP 1011; FCSA 61).

- Cotinga cayana (Linnaeus, 1766) spangled cotinga. - $\widehat{o}$ juv. LC (RNHMP 807; FCSA 191).

- Xipholena lamellipennis (Lafresnaye, 1839) white-tailed cotinga. - ad. NT (RNHMP 746; FCSA 73); (RNHMP 1002; FCSA 84).

Family Tityridae

- Schiffornis turdina (Wied, 1831) brown-winged mourner. - ad. LC (RNHMP 1010; FCSA 100).

Family Tyrannidae

- Rhynchocyclus olivaceus (Temminck, 1820) eastern olivaceous flatbill. - ad. LC (1014).

- Tyrannus caudifasciatus d'Orbigny, 1839 loggerhead kingbird. - ad. LC (RNHMP 741; FCSA 200).

- Knipolegus signatus (Taczanowski, 1874) Andean black-tyrant. - $q$ ad. LC (RNHMP 1009; FCSA 7).

Family Meliphagidae

- Anthochaera carunculata (Shaw, 1790) red wattlebird. - ad. LC (RNHMP 956).

- Prosthemadera novaeseelandiae G. R. Gray, 1840 tui. - ad. LC (RNHMP 819).

Family Oriolidae

- Oriolus xanthornus (Linnaeus, 1758) black-hooded oriole. - ad. LC (RNHMP 965; FCSA 174).

Family Pachycephalidae

- Colluricincla megarhyncha (Quoy \& Gaimard, 1830) little shrike-thrush. - ad. LC (RNHMP 1006).
Family Campephagidae

- Lalage melanoptera (Rüppell, 1839) black-headed cuckooshrike. - ad. LC (RNHMP 967; FCSA 49).

Family Artamidae

- Cracticus torquatus (Latham, 1801) grey butcherbird. - ad. LC (RNHMP 871; FCSA 89).

Family Vangidae

- Oriolia bernieri Geoffroy Saint-Hilaire, 1838 Bernier's vanga. - ad. VU (RNHMP 791; FCSA 54).

- Prionops caniceps (Bonaparte, 1850) red-billed helmetshrike. - ad. LC (RNHMP 742; FCSA 5); (RNHMP 784; FCSA 154).

Family Malaconotidae

- Laniarius barbarus (Linnaeus, 1766) yellowcrowned gonolek. - ad. LC (RNHMP 780; FCSA - ).

- Malaconotus gladiator (Reichenow, 1892) greenbreasted bush-shrike. - ad. VU (RNHMP 1013; FCSA 30).

Family Aegithinidae

- Aegithina lafresnayei lafresnayei (Hartlaub, 1844) great iora. - ad. LC (RNHMP 991).

Family Pityriaseidae

- Pityriasis gymnocephala gymnocephala (Temminck, 1835) Bornean bristlehead. - ad. NT (RNHMP 964; FCSA 43 A68).

Family Rhipiduridae

- Chaetorhynchus papuensis Meyer, 1874 drongo fantail. - ad. LC (RNHMP 1007).

Family Dicrurudae

- Dicrurus macrocercus (Vieillot, 1817) black drongo. - ad. LC (RNHMP 996; FCSA 91). 
The avian collection of the former Saint Augustine College of the Regional Natural History Museum in Plovdiv

Family Monarchidae

- Monarcha castaneiventris Verreaux, 1858 chestnut-bellied monarch. - ad. LC (RNHMP 778; FCSA 10 B17).

Family Corvidae

- Cyanocorax hafferi Cohn-Haft et al., 2013 Campina jay. - ad. NT (RNHMP 792; FCSA 60); (RNHMP 794; FCSA 81).

Family Paradisaeidae

- Paradisaea rubra Daudin, 1800 red bird-of-paradise. - ad. NT (RNHMP 796; FCSA 132).

- Paradisaea raggiana $\mathrm{P}$. L. Sclater, 1873 raggiana bird-of-paradise. - ad. LC (RNHMP 795).

Family Pycnonotidae

- Pycnonotus jocosus (Linnaeus, 1758) red-whiskered bulbul. - ad. LC (RNHMP 840; FCSA 184).

- Phyllastrephus xavieri (Oustalet, 1892) Xavier's greenbul. - ad. LC (RNHMP 1015; FCSA 50).

Family Polioptilidae

- Polioptila nigriceps (Baird, 1864) black-capped gnatcatcher. - ad. LC (RNHMP 995).

Family Buphagidae

- Buphagus africanus (Linnaeus, 1766) yellowbilled oxpecker. - ad. LC (RNHMP 1041).

Family Sturnidae

- Acridotheres grandis Moore, 1858 great myna. ad. LC (RNHMP 786; FCSA 128).

- Acridotheres tricolor (Daudin, 1800) grey-backed myna. - ad. CR (RNHMP 787; FCSA 46) (Fig. 4).

- Aplonis opaca (Kitttlitz, 1833) Micronesian starting. - ad. LC (RNHMP 783).

- Gracula religiosa Linnaeus, 1758 common hill myna. - ad. LC (RNHMP 811, 866; FCSA 8).

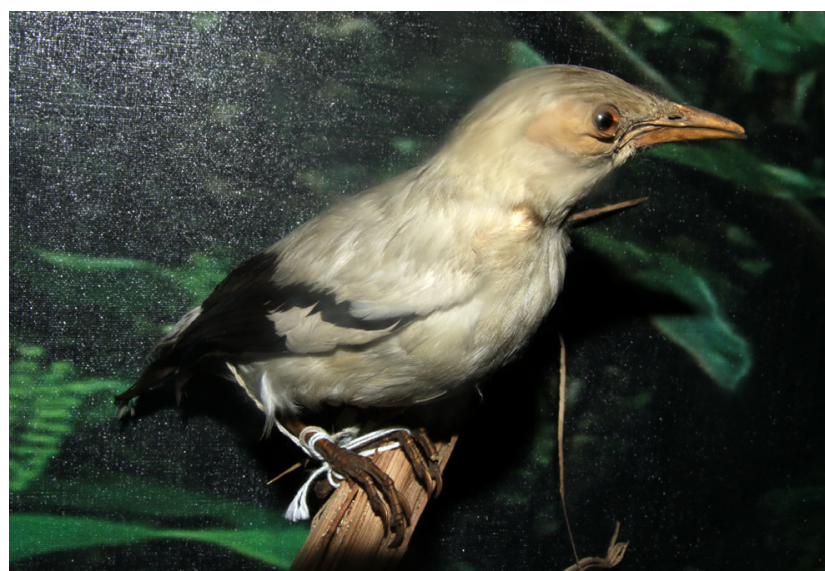

Fig. 4. Grey-backed myna (Acridotheres tricolor), RNHMP 787. Photograph: Ognyan Todorov.

Family Turdidae

- Turdus grayi Bonaparte, 1838 clay-colored thrush. - ad. LC (RNHMP 997, 1005; FCSA 82, 88).

- Turdus rufiventris Vieillot, 1818 rufous-bellied thrush. - ad. LC (RNHMP 992; FCSA 83).

- Turdus menachensis Ogilvie-Grant, 1913 Yemen thrush. - ad. NT (RNHMP 752; FCSA 14).

Family Muscicapidae

- Copsychus mindanensis (Boddaert, 1783) Philippine magpie-robin. - ad. LC (RNHMP 744; FCSA 217).

Family Bombycillidae

- Bombycilla garrulus (Linnaeus, 1758) Bohemian waxwing. - ad. LC (RNHMP 1030).

Family Irenidae

- Irena cyanogastra Vigors, 1831 Philippine fairybluebird. - ad. LC (RNHMP 841).

Family Ploceidae

- Anaplectes jubaensis (Sundewall, 1850) red-headed weaver. - ad. LC (RNHMP 1040; FCSA 226).

- Malimbus coronatus Sharpe, 1906 red-crowned malimbe. - ad. LC (RNHMP 849, 850; FCSA 198, 21). 
- Ploceus nigricollis (Vieillot, 1805) black-necked weaver. - ad. LC (RNHMP 972).

- Bubalornis albirostris (Vieillot, 1817) whitebilles buffalo-weaver. - juv. LC (RNHMP 999; FCSA 63).

- Bubalornis niger Smith, 1836 red-billed buffalo weaver. - 9 ad. LC (RNHMP 755; FCSA 96).

Family Viduidae

- Vidua orientalis (Heugin, 1870) sahel paradisewhydah. - ad. LC (RNHMP 758).

Family Fringillidae

- Linaria cannabina (Linnaeus, 1758) common linnet. - $q$ ad. LC (RNHMP 208).

- Euphonia pectoralis (Latham, 1801) chestnutbellied euphonia. - ad. LC (RNHMP 785); (RNHMP 848).

- Euphonia saturata (Cabanis, 1860) orangecrowned euphonia. - ad. LC (RNHMP 753, 845).

Family Emberizidae

- Plectrophenax nivalis (Linnaeus, 1758) snow bunting. - ad. LC (RNHMP 451).

Family Icteridae

- Cacicus cela (Linnaeus, 1758) yellow-rumped cacique. - ad. LC (RNHMP 737; FCSA 174); (RNHMP 966; FCSA 53).

- Cacicus pacificus (Sclater \& Salvin, 1864) pacific cacique. - ad. LC (RNHMP 788).

- Hypopyrrhus pyrohypogaster (De Tarragon, 1847) red-bellied grackle. - ad. VU (RNHMP 808; FCSA 80).

- Icterus galbula (Linnaeus, 1758) Baltimore oriole. - ad. LC (RNHMP 976; FCSA 204).

- Icterus icterus (Linnaeus, 1766) Venezuelan troupial. - ad. LC (RNHMP 735).

- Icterus spirius (Linnaeus, 1766) orchard oriole. ad. LC (RNHMP 993; FCSA 188).

- Leistes militaris (Linnaeus, 1758) red-breasted

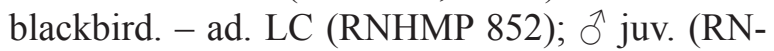
HMP 1004; FCSA 95).

- Molothrus oryzivorus (Gmelin, 1788) giant cowbird. - ad. LC (RNHMP 782; FCSA 63).
- Psarocolius decumanus (Pallas, 1769) crested oropendola. - ad. LC (RNHMP 736; FCSA 126).

- Quiscalus lugubris Swainson, 1838 Carib grackle. - ad. LC (RNHMP 817 FCSA; 93).

- Quiscalus mexicanus (Gmelin, 1788) great-tailed grackle. - $q$ ad. LC (RNHMP 1000; FCSA 38).

- Ptiloxena atroviolacea (d'Orbigny, 1839) Cuban blackbird. - juv. LC (RNHMP 998; FCSA 224).

Family Gardinalidae

- Habia rubica (Vieillot, 1817) red-crowned anttanager. - $q$ ad. LC (RNHMP 994; FCSA - ); $q$ ad. LC (RNHMP 851; FCSA 167).

Family Thraupidae

- Tangara punctata (Linnaeus, 1766) spotted tanager. - $q$ ad. LC (RNHMP 846).

- Tangara sayaca (Linnaeus, 1766) sayaca tanager. - ad. LC (RNHMP 745; FCSA 162).

- Sporophila lineola (Linnaeus, 1758) lined seedeater. - ad. LC (RNHMP 842).

- Dacnis lineata (Gmelin, 1789) black-faced dacnis. - ad. LC (RNHMP 949).

- Buthraupis montana (d'Orbigny \& Lafresnaye, 1837) hooded mountain-tanager. - ad. LC 757; FCSA 52).

- Ramphocelus carbo (Pallas, 1764) silver-beaked tanager. - 0 ad. LC (RNHMP 972; FCSA 228).

- Islerothraupis cristata (Linnaeus, 1766) flamecrested tanager. - $\widehat{o}$ ad. LC (RNHMP 1008).

\section{Conclusions}

A total of 193 species of birds of 81 families and 28 orders, represented by 205 specimens of dry mounted preserves survived until now (ca. two percent of the recent World avifauna).

According to their conservation status (IUCN), the species of this collection belong to five categories: LC -164 species, NT $-14, \mathrm{VU}-12, \mathrm{EN}-2$ and CR 1 species. Thus at present, over $15 \%$ of the species of the collection have a conservation status (NT to CR).

The most valuable world rarities are one critically endangered species - grey-backed myna (Acridotheres tricolor) and two endangered species - echo parakeet (Psittacula eques) and sooty albatross (Phoebetria fusca). 
The great majority of the specimens are unique for Bulgarian museum ornithological collections and represent the only specimens in the Bulgarian scientific collections at all. All this makes it a very important ornithological collection not only in Bulgaria, but also for the broader ornithological community abroad.

\section{Acknowledgments}

The authors are thankful to Dr Edward Dickinson (Brighton, UK), Roger Safford (London Borough upon Thames, UK) and Ron Demey (Hasselt, Belgium) for identification of some specimens from East Africa.

\section{References}

Angelov P. 1957 In the halls of the Natural History Museum in the town of Plovdiv. Priroda i znanie 4: 22-24. (In Bulgarian)

Boev Z. 1991 The ornithological collection of the National Museum of Natural History at the Bulgarian academy of sciences. Historia naturalis bulgarica 3: $37-48$.

Boev Z. 2003 The Ornithological Collections of the National Museum of Natural History of the Bul- garian Academy of Sciences: their History and Scientific Value. In: Rheinwald G. (ed.) Bird Collection in Europe. Bonner zoologische Beiträge $51(2 / 3)(2002): 157-163$.

del Hoyo J., Collar N. 2014 HBW and BirdLife International Illustrated Checklist of the Birds of the World. Volume 1: Non-passerines. Lynx Edicions, Barcelona, 903 pp.

del Hoyo J., Collar N. 2016 HBW and BirdLife International Illustrated Checklist of the Birds of the World. Volume 2: Passerines. Lynx Edicions, Barcelona, $1023 \mathrm{pp}$.

Georgiev D. 2017 The paleontological collection of the Regional Natural History Museum, Plovdiv. Bulletin of the Natural History Museum - Plovdiv 2: vii-xi.

Petrov T. 1983 Ornithological collection (Falconiformes and Pelecaniformes) of the Natural Science Museum in Plovdiv. Bulletin of the Museums of South Bulgaria 9: 55-58. (In Bulgarian).

Roselaar C.S. 2003 An inventory of major European bird collections. Bulletin of the British Ornithological Club 123A: 253-337.

Todorov O. 2016 The Regional Natural History Museum in Plovdiv. Bulletin of the Natural History Museum - Plovdiv 1: i-vii. 